\title{
Reversed Immunoglycomics Identifies $\alpha$-Galactosyl-Bearing Glycotopes Specific for Leishmania major Infection
}

Alba L. Montoya," Victoria M. Austin," Susana Portillo, Irodiel Vinales, Roger A. Ashmus, Igor Estevao, Sohan R. Jankuru, Yasser Alraey, Waleed S. Al-Salem, Álvaro Acosta-Serrano,* Igor C. Almeida,* and Katja Michael*

Cite This: JACS Au 2021, 1, 1275-1287

Read Online

ACCESS | Llll Metrics \& More | 回 Article Recommendations | sl Supporting Information

ABSTRACT: All healthy humans have high levels of natural anti$\alpha$-galactosyl ( $\alpha$-Gal) antibodies (elicited by yet uncharacterized glycotopes), which may play important roles in immunoglycomics: (a) potential protection against certain parasitic and viral zoonotic infections; (b) targeting of $\alpha$-Gal-engineered cancer cells; (c) aiding in tissue repair; and (d) serving as adjuvants in $\alpha$-Gal-based vaccines. Patients with certain protozoan infections have specific anti- $\alpha$-Gal antibodies, elicited against parasite-derived $\alpha$-Galbearing glycotopes. These glycotopes, however, remain elusive except for the well-characterized glycotope $\mathrm{Gal} \alpha 1,3 \mathrm{Gal} \beta 1,4 \mathrm{Glc}$ NAc $\alpha$, expressed by Trypanosoma cruzi. The discovery of new

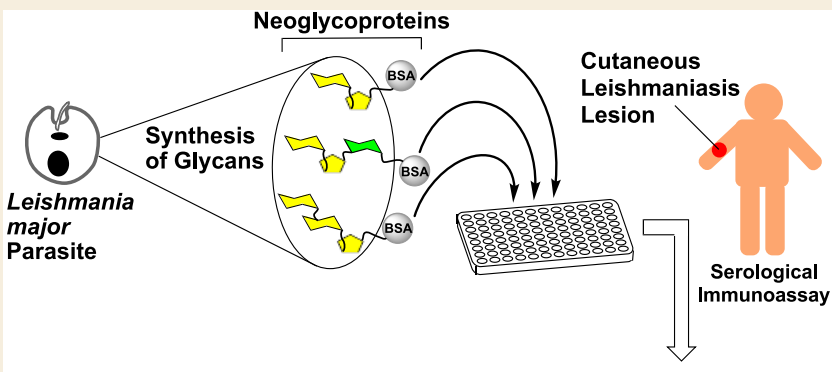
parasitic glycotopes is greatly hindered by the enormous structural

Diagnostic Biomarkers $\triangleleft$ Discovery of $\alpha$-Gal-Glycotopes diversity of cell-surface glycans and the technical challenges of classical immunoglycomics, a top-down approach from cultivated parasites to isolated glycans. Here, we demonstrate that reversed immunoglycomics, a bottom-up approach, can identify parasite species-specific $\alpha$-Gal-bearing glycotopes by probing synthetic oligosaccharides on neoglycoproteins. This method was tested here seeking to identify as-yet unknown glycotopes specific for Leishmania major, the causative agent of Old-World cutaneous leishmaniasis (OWCL). Neoglycoproteins decorated with synthetic $\alpha$-Gal-containing oligosaccharides derived from $L$. major glycoinositolphospholipids served as antigens in a chemiluminescent enzyme-linked immunosorbent assay using sera from OWCL patients and noninfected individuals. Receiver-operating characteristic analysis identified Galp $\alpha 1,3 \mathrm{Gal} f \beta$ and Galp $\alpha 1,3 \mathrm{Gal} f \beta 1,3$ Manp $\alpha$ glycotopes as diagnostic biomarkers for L. major-caused OWCL, which can distinguish with $100 \%$ specificity from heterologous diseases and L. tropica-caused OWCL. These glycotopes could prove useful in the development of rapid $\alpha$-Gal-based diagnostics and vaccines for OWCL. Furthermore, this method could help unravel cryptic $\alpha$-Gal-glycotopes of other protozoan parasites and enterobacteria that elicit the natural human anti- $\alpha$-Gal antibodies.

KEYWORDS: anti- $\alpha$-Gal antibodies, biomarkers, bioorganic chemistry, cutaneous leishmaniasis, glycoinositolphospholipids, Leishmania major, neoglycoproteins, oligosaccharide synthesis

\section{INTRODUCTION}

Old-World primates, including humans, do not express $\alpha$ galactose $(\alpha-\mathrm{Gal})$ in their glycoproteins due to the inactivation of the $\alpha 1,3$-galactosyltransferase gene in ancestral Old-World primates 20-30 million years ago. ${ }^{1}$ Therefore, humans are capable of producing anti- $\alpha$-galactosyl antibodies (anti- $\alpha$-Gal Abs) against $\alpha$-Gal-bearing antigens. Due to continuous stimulation by antigenic $\alpha$-Gal-containing lipopolysaccharides of enterobacteria, ${ }^{2}$ high titers of anti- $\alpha$-Gal Abs are maintained life-long in normal human serum (NHS). ${ }^{3}$ The exact structures of the glycotopes that collectively elicit these anti- $\alpha$-Gal Abs are yet to be identified and structurally characterized. While it is unknown whether natural anti- $\alpha$-Gal Abs have a distinct physiological purpose, they are important players in immunoglycomics due to their cross-reactivity with other $\alpha$ Gal-containing glycans. For example, their cross-reactivity with the Galili trisaccharide, $\operatorname{Gal} \alpha 1,3 \mathrm{Gal} \beta 1,4 \mathrm{GlcNAc} \beta$, which is expressed by all mammals other than Old-World primates, ${ }^{1}$ is responsible for the hyperacute rejection of porcine xenografts. ${ }^{4}$ On the other hand, they are likely to interfere with the transmission of $\alpha$-Gal-containing enveloped animal viruses to humans, thus protecting humans from zoonotic viral diseases. ${ }^{5}$ The binding of natural anti- $\alpha$-Gal Abs from NHS (or from $\alpha 1,3$-galactosyltransferase-knockout mice or pigs) to Gal $\alpha 1,3$ -

Received: May 10, 2021

Published: July 12, 2021 
$\mathrm{Gal} \beta$-containing glycans has been exploited in a number of biomedical contexts. ${ }^{5}$ Examples include the anti- $\alpha$-Gal Absmediated recruitment of macrophages by $\alpha$-Gal nanoparticles in wound healing and tissue repair, the decoration of cancer cells and viral and bacterial pathogens with $\alpha$-Gal-containing bifunctional molecules to increase their antigenicity and cause their destruction by the immune system, ${ }^{5-7}$ and the development of $\alpha$-Gal-expressing tumor cells as anticancer vaccines. Furthermore, $\alpha$-Gal residues of glycoengineered protein- and virus-based vaccines form immunocomplexes with anti- $\alpha$-Gal Abs, serving as adjuvants leading to enhanced uptake by antigen-presenting cells. ${ }^{9}$ The natural (or NHS) anti- $\alpha$-Gal Abs are also known to cross-react with $\alpha$-Gal-expressing protozoa, i.e., some Leishmania spp., ${ }^{10,11}$ Trypanosoma ${ }_{\text {cruzi }}{ }^{10,12,13}$ Trypanosoma brucei, ${ }^{14}$ and Plasmodium falciparum, ${ }^{15-17}$ the causative agents of leishmaniasis, Chagas disease (CD), African trypanosomiasis, and malaria, respectively. These antibodies could be the first line of defense to fend off the establishment of parasitic infections in Old-World primates. ${ }^{5}$ In patients with these infectious diseases, parasitic $\alpha$-Gal-bearing glycotopes elicit specific anti- $\alpha$-Gal Abs that exceed the NHS anti- $\alpha$-Gal Abs in both concentration and binding strength and, ultimately, in specificity. ${ }^{10,12,15,18}$ Knowledge of these glycotopes could open doors for biomedical applications such as diagnostics and vaccine development. Unfortunately, the exact structures of these glycotopes are unknown, with the exception of the well-established T. cruzi glycotope Gal $\alpha 1,3 \mathrm{Gal} \beta 1,4 \mathrm{GlcNAc} \alpha$, which makes up to $\sim 10 \%$ of the parasite's $\alpha$-Gal-containing mucin O-glycans. ${ }^{18}$ The identification of parasitic $\alpha$-Gal glycotopes by classic immunoglycomics requires cultivation of large amounts of parasites (sometimes, unfeasible depending upon the parasite life-cycle stage), and isolation and analysis of the cell surface glycans, which require a vast array of analytical methods, such as glycoproteomics, enzymatic and/or chemical release and fluorescent labeling of glycans, glycoprofiling by various highresolution mass spectrometry (HR-MS)-based approaches, and nuclear magnetic resonance (NMR), among others. ${ }^{19-21}$ This top-down approach is further complicated by the enormous structural diversity of parasite glycocalyces. ${ }^{22}$ To get around these technical challenges and limitations, we pursued a bottom-up approach, akin to reversing the classical immunoglycomics approach: based on known or potential parasitic $\alpha$ Gal-glycotopes, glycans of different sizes are synthesized, coupled to a carrier protein, and their antigenicity is interrogated with sera from patients. Here, we show that this reversed immunoglycomics approach can lead to the discovery of novel parasitic $\alpha$-Gal glycotopes. We selected Old-World cutaneous leishmaniasis (OWCL) as a proof of concept for two reasons: (a) L. major, one of the causative agents of OWCL, abundantly expresses $\alpha$-Gal-bearing type- 2 glycoinositolpholipids (GIPLs) whose structures are known ${ }^{23}$ and are likely to contain immunodominant glycotopes; (b) the discovery of $L$. major glycotopes would be impactful because they could serve as biomarkers (BMKs) for OWCL, for which currently no molecular BMKs exist.

Cutaneous leishmaniasis (CL) is a vector-borne infectious disease caused by different species of the protozoan parasite Leishmania, and is transmitted by infected female sandflies. ${ }^{24}$ OWCL is endemic to the Mediterranean basin, Middle East, and Central Asia, where approximately 700000 new infections are reported annually. ${ }^{25}$ While $\mathrm{CL}$ is usually not lifethreatening, it causes large disfiguring skin ulcers, often associated with secondary infections, that may take months to years to heal, and the scarring often leads to social stigma and depression. ${ }^{26}$ In Northern Africa and the Middle East, the two most prevalent OWCL-causing Leishmania species are $L$. major and L. tropica. ${ }^{27}$ In the absence of a protective OWCL vaccine, the only feasible way to control the disease is through chemotherapy. ${ }^{28}$ However, treatment responses vary depending on the infecting parasite species. ${ }^{24,29}$ For example, topical azoles/fusidic acid ointments are effective for treating OWCL caused by L. major but are ineffective for L. tropica. ${ }^{29}$ This underscores the need for a species-specific diagnosis of OWCL. $^{24}$

OWCL is commonly diagnosed by inspection of skin lesions, but the lesions closely resemble other skin conditions, such as atopic dermatitis (eczema), skin cancer, and Hansen's disease. ${ }^{28,30}$ Other diagnostic methods include the detection of Leishmania from skin lesions by microscopy, histopathology (amastigotes), and in culture (promastigotes), as well as serology using whole parasite lysate as the antigen. ${ }^{24,20}$ However, the limited sensitivity and specificity of these methods do not always accurately diagnose OWCL ${ }^{20}$ and cannot differentiate between infecting Leishmania species. ${ }^{24}$ The polymerase chain reaction has an extraordinary sensitivity and can differentiate between species, but it is expensive, technologically demanding, and often unavailable in clinical laboratories in developing countries, ${ }^{28,31}$ where an affordable, species-specific diagnostic test is needed the most. To put this into further context, the continuing unrest in the Middle East, particularly in Syria, Afghanistan, and Iraq, has resulted in a massive displacement of individuals who are potentially infected with L. major or L. tropica. ${ }^{32}$ Many of these individuals live in CL-endemic refugee settings and areas where diagnostic capabilities are limited. This population group would particularly benefit from a robust, diagnostic species-specific OWCL assay to inform best drug treatment options.

\section{RESULTS AND DISCUSSION}

The glycocalyx of Leishmania parasites is predominantly composed of GIPLs, as well as lipophosphoglycans (LPGs) and glycosylphosphatidylinositol (GPI)-anchored proteins, such as GP63 and proteophosphoglycans (PPGs). ${ }^{23,33-36} \mathrm{~L}$. major synthesizes highly abundant type-2 GIPLs (GIPL-1, GIPL-2, and GIPL-3), which contain a $\beta$-galactofuranosyl ( $\beta$ Galf) (GIPL- 1$)$ or $\alpha$-galactopyranosyl $(\alpha$-Galp) (GIPL- 2 and GIPL-3) residue at their terminal, nonreducing ends. ${ }^{23}$ There is a considerable body of evidence that these unusual sugars expressed in L. major and in other pathogens, such as T. cruzi, fungi, and enterobacteria, are highly immunogenic to human hosts because they are either cryptic or not normally expressed $(\alpha$-Gal $p)$, or entirely absent $(\beta$-Galf $)$ on human cells. ${ }^{23,37-44}$ On the other hand, the main GIPLs of L. tropica belong to the $\alpha$-mannose-terminating GIPLs iM2, iM3, and iM4. ${ }^{34} \mathrm{We}$ hypothesized that terminal oligosaccharide partial structures of type-2 GIPLs of L. major may be immunodominant glycotopes that are serologically exploitable as diagnostic BMKs specifically for OWCL caused by L. major and distinguishable from OWCL caused by L. tropica, as well as from non-OWCL dermatological (heterologous) diseases, such as atopic dermatitis (eczema), and bacterial and fungal infections. To address this hypothesis, specific $L$. major-derived glycan partial structures needed to be synthesized and immunologically evaluated. 


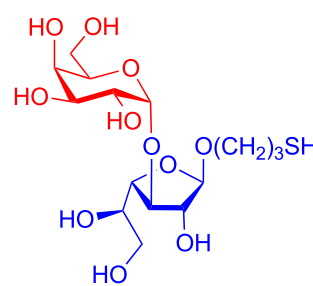

$\mathbf{G}_{\text {SH }}$

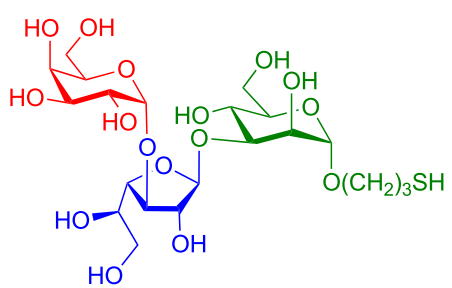

$\mathrm{G}^{3} \mathbf{O}_{\mathrm{SH}}$

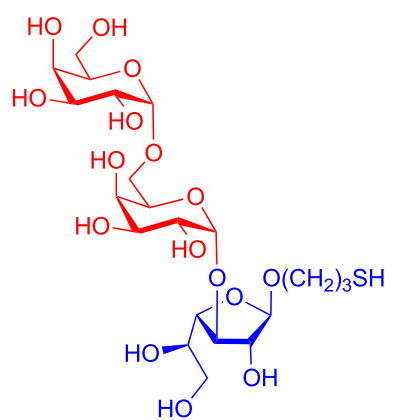

$\mathbf{G}^{2} 8_{\text {SH }}$

Figure 1. Target 3-thiopropyl glycosides of Galp $\alpha 1,3 \mathrm{Gal} f \beta\left(\mathbf{G}_{\mathbf{S H}}\right)$, Galp $\alpha 1,3 \mathrm{Gal} f \beta 1,3 \mathrm{Man} \alpha\left(\mathbf{G}_{\mathbf{S H}}\right)$, and Galp $\alpha 1,6 \mathrm{Gal} p \alpha 1,3 \mathrm{Gal} f \beta\left(\mathbf{G} 28_{\mathrm{SH}}\right)$ derived from type-2 GIPL-2 and GIPL-3 of L. major.

Scheme 1. Synthesis of 3-Thiopropyl Glycosides $\mathrm{G} 27_{\mathrm{SH}}, \mathrm{G} 30_{\mathrm{SH}}$, and $\mathrm{G} 28_{\mathrm{SH}}$

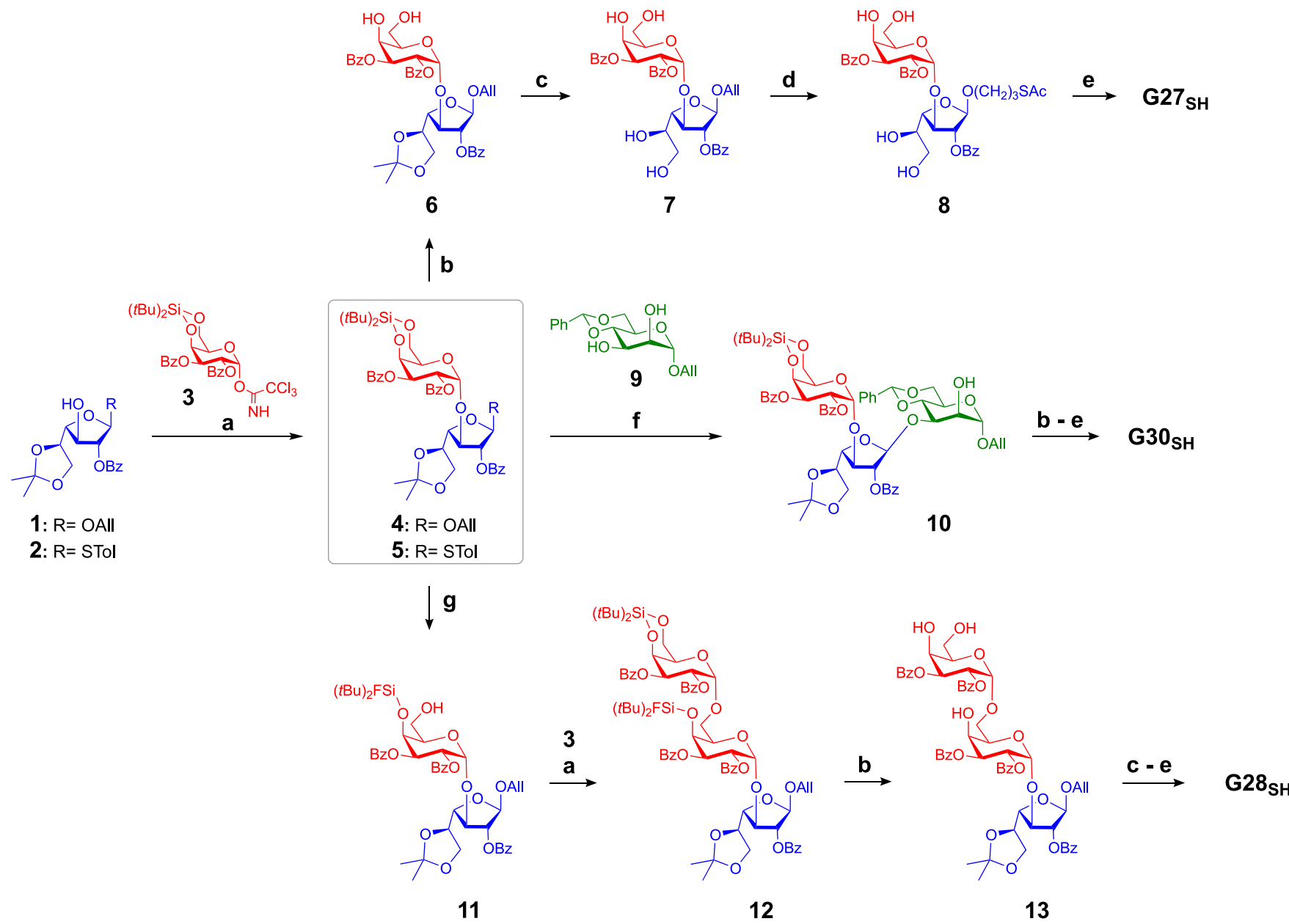

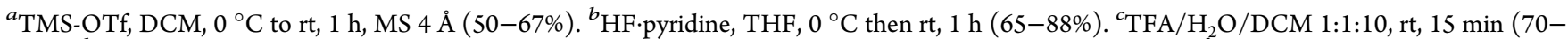
$90 \%) .{ }^{d} \mathrm{AcSH}, \mathrm{AIBN}$ or DPAP, THF, UV light $(350 \mathrm{~nm}), 6-12 \mathrm{~h}(85-93 \%) .{ }^{e} \mathrm{NaOMe}, \mathrm{MeOH}, \mathrm{rt}, 2 \mathrm{~h}$ (quant.). ${ }^{\circ} \mathrm{NIS}, \mathrm{AgOTf}, \mathrm{DCM}, 0{ }^{\circ} \mathrm{C}$ to rt, 45 min, MS $4 \AA$ (47\%). ${ }^{g}$ HF.pyridine (1.2 equiv), THF, $0{ }^{\circ} \mathrm{C}, 6 \mathrm{~h}$ or HF.pyridine (excess), THF, $0{ }^{\circ} \mathrm{C}, 2 \mathrm{~h}(60 \%)$.

Here, we present reversed immunoglycomics as a combined chemical and serological approach to discover specific glycan BMKs suitable for the diagnosis of L. major infection and for the distinction from L. tropica infection and heterologous diseases, using synthetic cell surface glycans of the parasite. Instead of isolating glycoconjugates from large-scale cultivated parasites and studying their glycoimmunology, we have reversed this classical immunoglycomics approach by probing synthetic partial structures of known cell surface glycans for antibody responses by the highly sensitive chemiluminescent enzyme-linked immunosorbent assay (ELISA). Our previous studies showed that the sera of OWCL patients from Saudi Arabia contain elevated levels of anti- $\alpha$-Gal IgG antibodies ${ }^{45}$ that partially recognize simple $\alpha$-Gal-containing saccharides, but a specific $\alpha$-Gal BMK remained elusive. ${ }^{46}$ Therefore, we shifted our focus to $\alpha$-galactopyranose- and $\beta$-galactofuranosecontaining saccharides of the terminal, nonreducing glycan portions of type-2 GIPLs of L. major. The synthesis of a 
Scheme 2. Conjugation of Glycans and Maleimide Derivatized BSA 14 to Produce NGPs

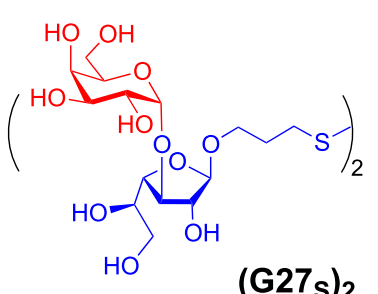
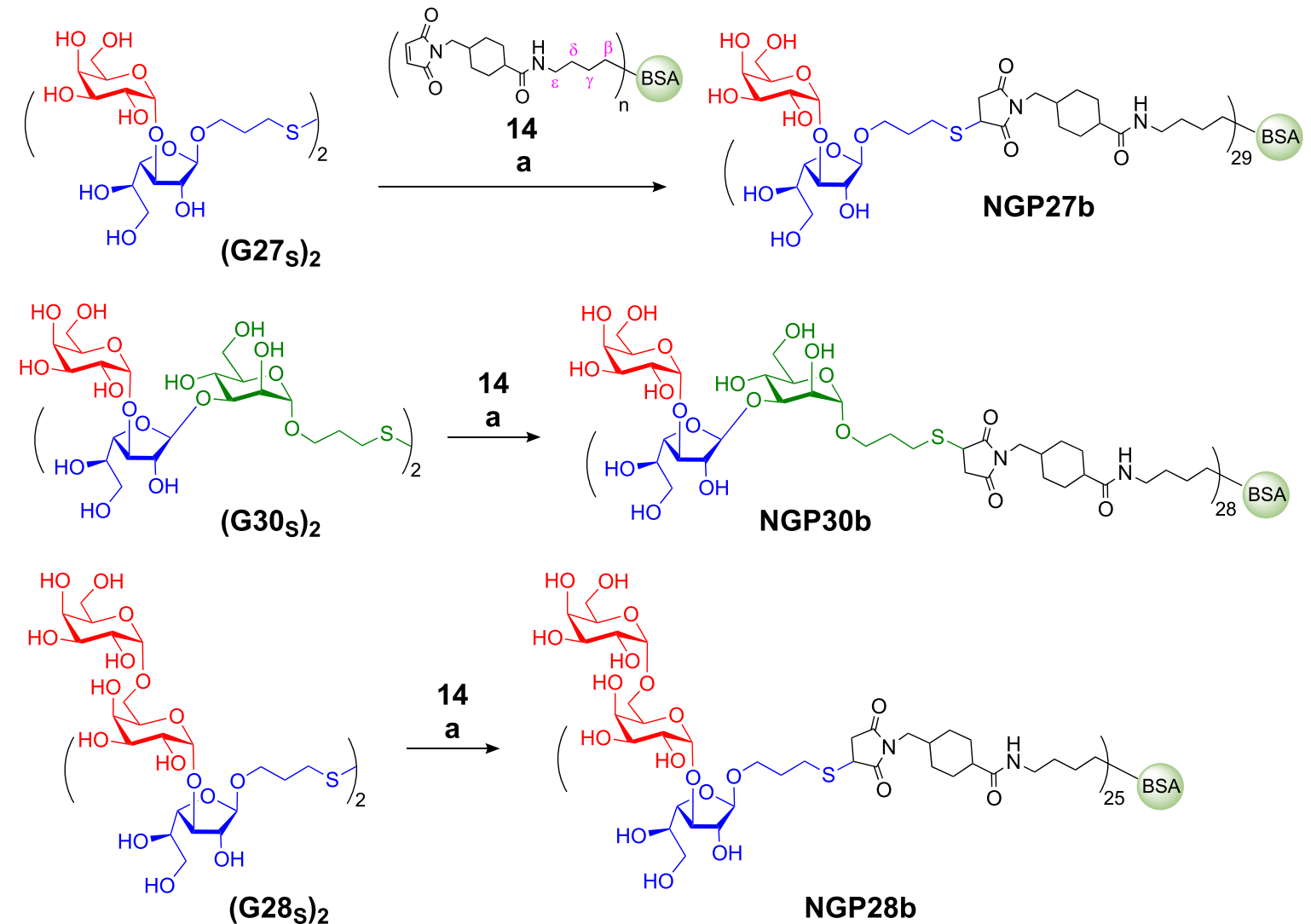

NGP28b

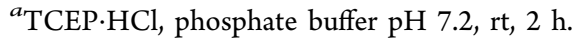

terminal trisaccharide of type-2 GIPLs and a heptasaccharide present in LPGs of Leishmania parasites has been reported. ${ }^{47,48}$ Here, we have developed original syntheses for three type-2 GIPL partial structures with a chemical handle at the reducing end, allowing for conjugation to a carrier protein. Specifically, 3-thiopropyl glycosides of the GIPL-2-derived glycans Galp $\alpha 1,3 \mathrm{Gal} f \beta\left(\mathbf{G}_{2} 7_{\mathrm{SH}}\right)$ and $\mathrm{Gal} p \alpha 1,3 \mathrm{Gal} f \beta 1,3 \mathrm{Man} p \alpha$ $\left(\mathbf{G 3 0}_{\mathrm{SH}}\right)$, as well as the GIPL-3-derived glycan Galp $\alpha 1,6 \mathrm{Gal}$ $p \alpha 1,3 \mathrm{Gal} f \beta\left(\mathbf{G}^{2} \mathbf{8}_{\mathrm{SH}}\right)$, were synthesized (Figure 1). Conjugation of these oligosaccharides to bovine serum albumin (BSA) produced neoglycoproteins (NGPs) which, unlike the oligosaccharides by themselves, adhere effectively to ELISA microplates. These NGPs served as antigens to be evaluated by chemiluminescent ELISA, ${ }^{45,49}$ using sera of OWCL patients from Saudi Arabia with confirmed L. major or L. tropica infections.

Our synthetic strategy for all three oligosaccharides $\mathbf{G 2 7} 7_{\mathrm{SH}}$, $\mathbf{G} 30_{\mathrm{SH}}$, and $\mathbf{G 2 8} \mathbf{S}_{\mathrm{SH}}$ relied on (a) the stereoselective $\alpha$ galactosylation using Kiso's 4,6-di-tert-butylsilylene-galactosyl trichloroacetimidate donor; $^{50,51}$ (b) an orthogonal protecting group strategy primarily based on acyl, acetal, and silyl groups; and (c) the use of 3-thiopropyl glycosides for the conjugation to maleimide-derivatized BSA. ${ }^{52,53}$ The synthesis of GIPL-3derived trisaccharide $\mathbf{G} \mathbf{2} \mathbf{8}_{\mathrm{SH}}$ also included a regioselective ringopening of the 4,6-O-di-tert-butylsilyene (DTBS) moiety to gain access to a disaccharide acceptor in a single step.

The synthesis of the three target oligosaccharides $\mathbf{6 2 7} 7_{\mathrm{SH}}$, $\mathbf{G} 30_{\mathrm{SH}}$, and $\mathbf{G} 28_{\mathrm{SH}}$ started with the preparation of the monosaccharide building blocks $\mathbf{1},{ }^{54} \mathbf{2}^{55-57}$ and $3 .^{50}$ In order to construct 3-thiopropyl $\alpha$-D-galactopyranosyl-( $1 \rightarrow 3)$ -
$\beta$-D-galactofuranoside $\mathbf{G 2 7}{ }_{\mathrm{SH}}$ (Scheme 1 ), Galf $\beta$ acceptor $\mathbf{1}^{54}$ was glycosylated with Galp $\alpha$ donor $3^{50}$ to furnish the fully protected disaccharide 4 in $65 \%$ yield. The silylene group was removed using HF-pyridine complex to give compound $\mathbf{6}$ in $65 \%$ yield, and then, the isopropylidene group was hydrolyzed with aqueous trifluoroacetic acid (TFA) to afford allyl glycoside 7 in $90 \%$ yield. The radical addition of thioacetic acid $(\mathrm{AcSH})$ to 7 in dry THF furnished thioester 8 in $85 \%$ yield. Complete deacylation under Zemplén conditions provided the target disaccharide $\mathbf{G} 27_{\mathrm{SH}}$ quantitatively, which oxidized to disulfide $\left(\mathbf{G 2 7} \mathbf{S}_{\mathbf{S}}\right)_{2}$.

The trisaccharide 3-thiopropyl $\alpha$-D-galactopyranosyl-(1 $\rightarrow 3)$ $\beta$-D-galactofuranosyl-( $1 \rightarrow 3)$ - $\beta$-D-mannopyranoside $\mathbf{G} 3 \mathbf{0}_{\mathrm{SH}}$ was synthesized by two consecutive glycosylation steps. First, the Galf $\beta$ acceptor $2^{55-57}$ was glycosylated with Galp $\alpha$ donor $3^{54}$ with high stereoselectivity to produce disaccharide 5 in $67 \%$ yield. This donor was used to regioselectively glycosylate mannosyl acceptor $9^{58}$ to yield trisaccharide 10 in $47 \%$ yield. A similar regioselective glycosylation was previously reported on an analogous mannosyl acceptor. ${ }^{48}$ Afterward, the silylene protecting group was removed with HF-pyridine complex, and without purification, both the benzylidene and isopropylidene groups were removed by acid-catalyzed hydrolysis to give compound $\mathbf{S} 7$ in $48 \%$ yield over two steps. The radical addition of AcSH to the allyl glycoside was achieved in $85 \%$ yield to provide thioester S8. ${ }^{54}$ Finally, global removal of all ester groups under Zemplén conditions furnished the desired trisaccharide $\mathbf{G} 3 \mathbf{0}_{\mathrm{SH}}$ quantitatively (Scheme 1), which oxidized to disulfide $(\mathbf{G 3 0})_{2}$. 
a)

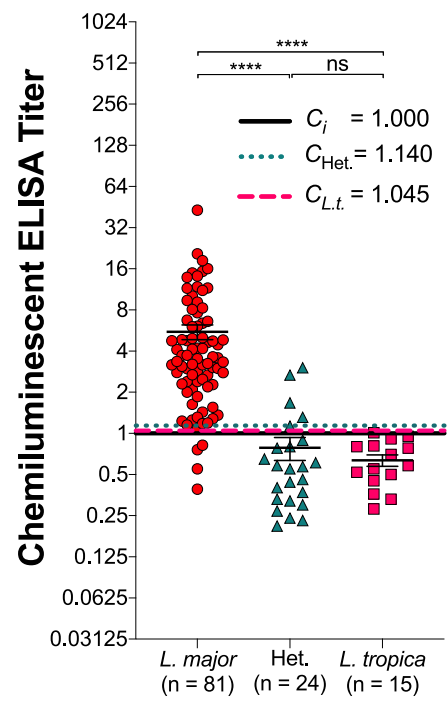

b)

L. major

vs.

Heterologous

L. major

vs.

L. tropica

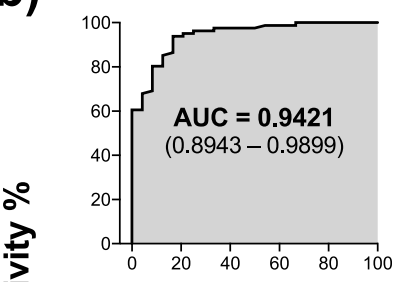

NGP27b

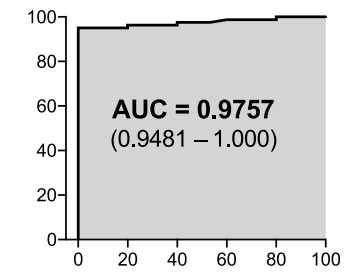

c)

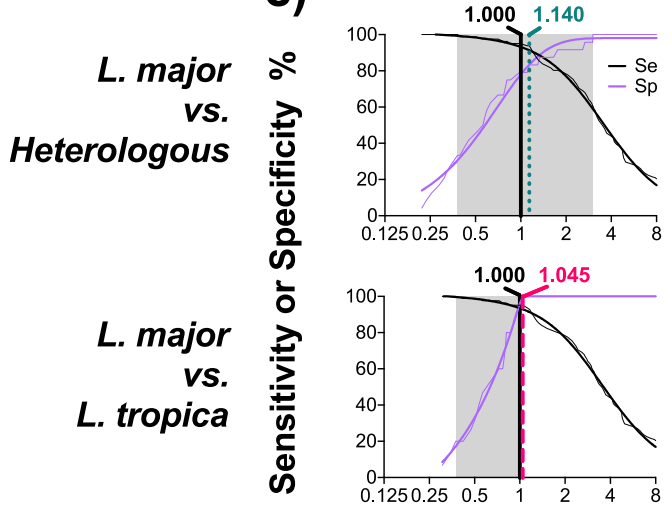

NGP28b

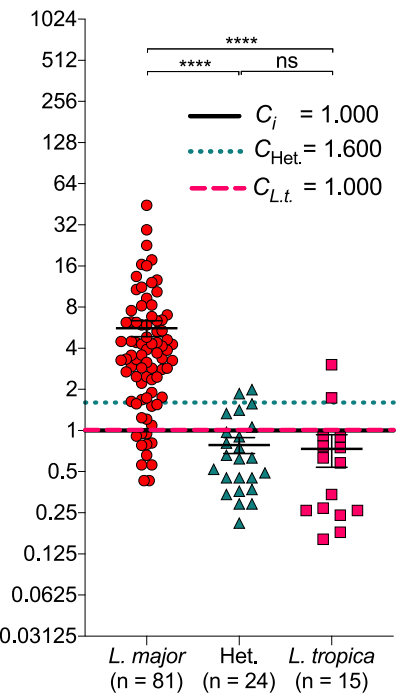

Serum Panel
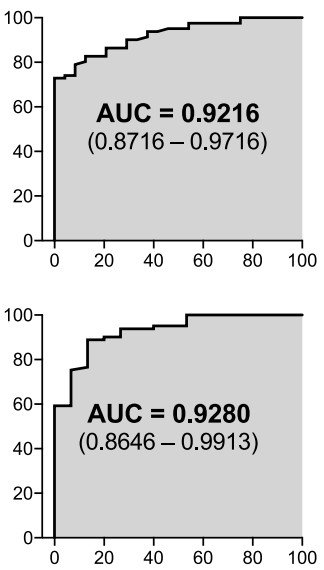

$100 \%$ - Specificity $\%$
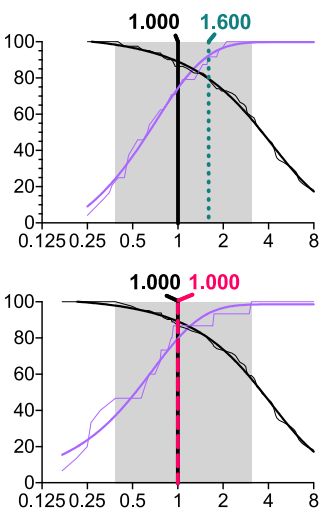

Cutoff value
NGP30b
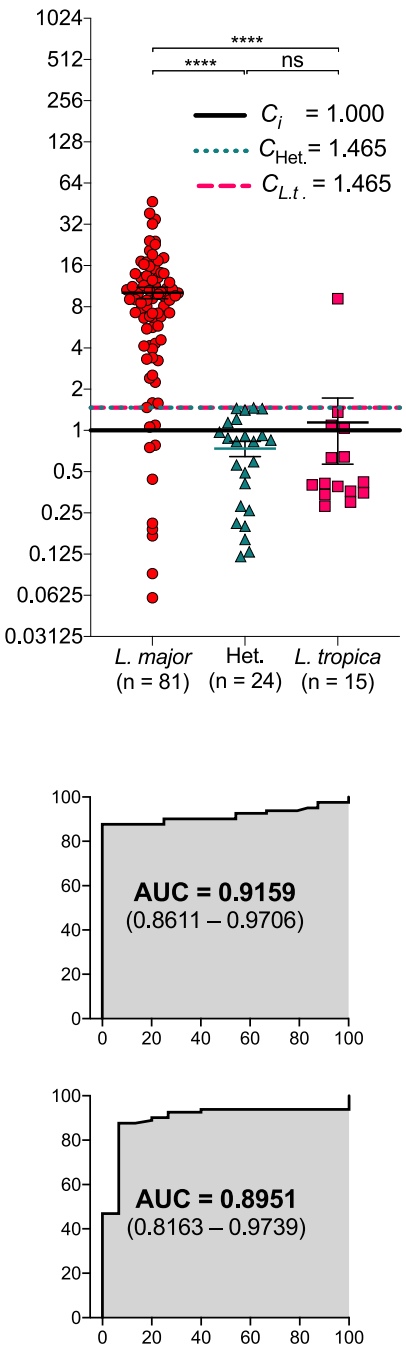
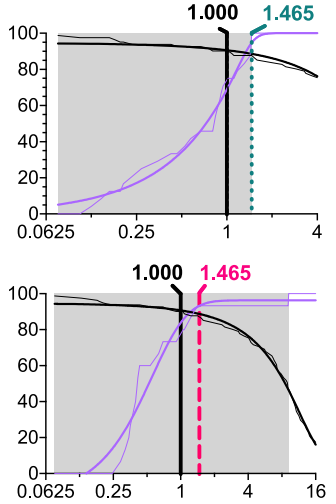

Figure 2. Chemiluminescent ELISA reactivity of NGP27b, NGP28b, and NGP30b with sera from individual patients with L. major or L. tropica infection or with heterologous disease. Sera (at 1:800 dilution) from individual patients with active L. major or L. tropica infection or with heterologous disease (with other skin conditions) were evaluated against NGP27b, NGP28b, and NGP30b, each at $25 \mathrm{ng} /$ well. Chemiluminescent ELISA was performed as described in the Supporting Information ("Chemiluminescent ELISA"). (a) Grouped scatter plot analysis of sera from $L$. major or L. tropica infections or heterologous diseases (Het.) with NGP27b, NGP28b, or NGP30b. The $Y$-axis values are in log2 scale. $C_{i}$, initial cutoff value (titer $=1.000)$, calculated as described in the Supporting Information. $C_{L . t}$, adjusted cutoff value, calculated based on the ROC and TG-ROC curve analysis data $(b, c)$, for the comparison between sera from L. major vs L. tropica infections. $C_{\text {Het., }}$ adjusted cutoff value for the comparison between sera from L. major infection vs heterologous diseases. Statistical analysis: nonparametric Mann-Whitney test. Significance 
Figure 2. continued

level: $p<0.05$. $* * * *, p<0.0001$; ns, nonsignificant. (b) Receiver-operating characteristic (ROC) curves for NGP27b, NGP28b, and NGP30b, comparing the reactivity of sera from L. major vs L. tropica infections (top row) or sera from L. major infections vs heterologous diseases (bottom row), using the data depicted in the scatter plots (a). AUC, area under the curve, is indicated (gray area). In parentheses, $95 \%$ confidence interval values are indicated. (c) A TG-ROC curve analysis was performed by plotting the ROC data (b) for sensitivity (Se) and specificity (Sp), as described by Greiner et al. ${ }^{64}$ Shaded area indicates the cutoff value interval where Se or Sp could reach $100 \%$. The Se (black) and Sp (purple) raw data points are represented as fine lines, whereas the fitted data are indicated as thick lines. Vertical black line, original titer cutoff value $\left(C_{\mathrm{i}}=\right.$ 1.000); vertical dotted green line, adjusted cutoff value for the comparison of L. major infections vs heterologous diseases; vertical dashed red line, adjusted cutoff value for the comparison of $L$. major vs $L$. tropica infections. The adjusted cutoff values are indicated in green or red.

The trisaccharide 3-thiopropyl $\alpha$-D-galactopyranosyl-(1 $\rightarrow 6)$ $\alpha$-D-galactopyranosyl- $(1 \rightarrow 3)-\beta$-D-galactofuranoside $\mathbf{G} 28_{\text {SH }}$ was synthesized from disaccharide 4 . We found by serendipity that the 4,6-O-DTBS ring of disaccharide 4 can be regioselectively opened with HF.pyridine rendering only position 4 of the $\alpha$ Gal residue protected as a di-tert-butylfluorosilyl ether and OH-6 unprotected. A similar regioselective ring-opening of a 3,5-O-DTBS group of a galactofuranoside with tetrabutylammonium fluoride (TBAF) had been reported; ${ }^{59}$ however, the 4,6-O-DTBS group of a galactopyranoside resists reaction with TBAF, even when used in large excess. Conversely, 1.2 equiv of HF.pyridine complex at $0{ }^{\circ} \mathrm{C}$ opened the silylene ring of disaccharide 4 within 6 h. The reaction time could be reduced to only $2 \mathrm{~h}$ by using HF.pyridine in excess at $0{ }^{\circ} \mathrm{C}$ and immediate quenching with aqueous saturated $\mathrm{NaHCO}_{3}$ solution. The resulting disaccharide 11 was obtained in $60 \%$ yield, and we envisioned it to be used as a glycosyl acceptor. One concern was that the bulky silyl group at position 4 could potentially hinder the glycosylation at OH-6; however, donor $3^{50}$ was able to glycosylate acceptor 11 to provide the fully protected trisaccharide 12, with an exclusive $\alpha$-stereoselectivity in an acceptable yield of $50 \%$. Subsequent treatment with HF. pyridine complex in excess from $0{ }^{\circ} \mathrm{C}$ to room temperature (rt) removed the silyl and silylene groups to produce trisaccharide 13 in $88 \%$ yield. Acid-catalyzed hydrolysis of the isopropylidene group of $\mathbf{1 3}$ produced compound S5 in $77 \%$ yield, and the thioester derivative S6 was prepared by the radical addition of AcSH in $93 \%$ yield. ${ }^{54}$ Global deprotection under Zemplén conditions furnished the target 3-thiopropyl trisaccharide $\mathbf{G} 28_{\mathrm{SH}}$ quantitatively (Scheme 1), which oxidized to disulfide $\left(\mathbf{G} 28_{\mathbf{S}}\right)_{2}$.

Reduction of the disulfides $\left(\mathbf{G} 27_{\mathrm{S}}\right)_{2},\left(\mathbf{G} 28_{\mathrm{S}}\right)_{2}$, and $\left(\mathbf{G} 30_{\mathrm{S}}\right)_{2}$ with TCEP. $\mathrm{HCl}$ and conjugation to commercial maleimidederivatized BSA 14 produced the conjugates NGP27b, NGP28b, and NGP30b, respectively (Scheme 2). The average number of glycans conjugated per BSA molecule was estimated by matrix-assisted laser/desorption ionization time-of-flight mass spectrometry (MALDI-TOF-MS). Using the known masses of glycans and linker, the payloads $(n=29,25$, and 28) were calculated by subtracting the average mass of BSA from the average masses of NGP27b, NGP28b, and NGP30b, respectively (Figures $\mathrm{S} 1-\mathrm{S} 3$ ). ${ }^{54}$

With the NGP antigens in hand, IgG antibody responses of serum samples from patients with acute L. major or L. tropica infection, confirmed by dermatological examination, microscopic analysis of lesion aspirate, and PCR-restriction fragment length polymorphism-based analysis using the rDNA internal transcribed spacer 1 (rDNA-ITS1) (ITS1-PCR-RFLP), ${ }^{60}$ could now be studied by chemiluminescent ELISA. The serum samples of patients had been collected from 81 individuals with active $L$. major infection and 15 individuals with active L. tropica infection, from the areas Al-Ahsa and Asir in Saudi Arabia, where L. major and L. tropica infections are endemic. $^{29}$ In addition, pooled sera of 10 healthy individuals, designated normal human serum (NHS), from England, UK, as well as sera from 24 individuals with heterologous diseases from the Al-Ahsa governorate, Saudi Arabia, were also integrated in the study (more detail about the study population and diagnostic methods is provided in the Supporting Information, "Ethics Statement and Cohort Description, Chemiluminescent ELISA”).

The heterologous sera were from patients who had skin conditions other than CL. Therefore, these sera represent a real-life, non-CL negative control group, relevant to what a physician may encounter, from which a useful CL BMK must be able to distinguish. Since all NHS contains anti- $\alpha$-Gal antibodies directed against $\alpha$-Gal-containing lipopolysaccharides of Gram-negative enterobacteria, ${ }^{61,62}$ a small amount of cross-reactivity between NHS anti- $\alpha$-Gal antibodies and the $L$. major-derived glycostructures might be expected, as previously observed with purified L. major type-2 GIPL-1, GIPL-2, and GIPL-3. ${ }^{39}$ In order to ensure that chemiluminescent ELISA responses were not a result of antibody binding to BSA or the cross-linker, 2-MEb, obtained by conjugating 2-mercaptoethanol (2-ME) to maleimide-derivatized BSA 14 (Figure S1c), was used as a negative control antigen. In order to identify a suitable protocol for the chemiluminescent ELISA, serum pools were prepared and cross-titrated against different concentrations [ng/well] of the three NGPs and 2-MEb. Figure S5 shows that the L. major serum pool at different dilutions (1:400-1:3200) exhibited a high IgG antibody reactivity to NGP27b, NGP28b, and NGP30b, at different concentrations $(3-400 \mathrm{ng} /$ well), whereas the L. tropica and NHS (healthy) serum pools showed a significantly lower antibody reactivity at different serum dilutions and antigen concentrations. The cross-titration experiment revealed that a serum dilution of 1:800 and an antigen loading of $25 \mathrm{ng} /$ well exhibited the highest differential antibody reactivity between the $L$. major-positive serum pool and serum pools from $L$. tropica-positive or healthy individuals. Under these conditions, the reactivity ratios between $L$. major and healthy serum pools were $\sim 6$-, $\sim 9$-, and $\sim 56$-fold for NGP27b, NGP28b, and NGP30b, respectively. Comparing L. major vs L. tropica serum pools, the reactivity ratios were $\sim 90-, \sim 9$-, and $\sim 16$-fold for NGP27b, NGP28b, and NGP30b, respectively. As expected, the negative control $\mathbf{2 - M E b}$ showed no or little reactivity with all pooled sera, indicating that no significant antibody binding occurred to BSA or the cross-linker (Figure S5).

Next, NGP27b, NGP28b, and NGP30b were tested as potential BMKs for the accurate diagnosis of L. major infections and, in particular, differentiating these from heterologous diseases, which is a major issue in clinical settings in some endemic and nonendemic regions, where there is a high migration of CL patients from affected areas. 
Table 1. Reactivity of Sera from L. major Infections, Heterologous Diseases, and L. tropica Infections with NGP27b, NGP28b, and NGP30b

\begin{tabular}{|c|c|c|c|c|c|c|c|}
\hline \multirow[b]{2}{*}{ infection/disease } & \multirow[b]{2}{*}{$n$} & \multicolumn{2}{|c|}{ NGP27b } & \multicolumn{2}{|c|}{ NGP28b } & \multicolumn{2}{|c|}{ NGP30b } \\
\hline & & positive & negative & positive & negative & positive & negative \\
\hline \multicolumn{8}{|c|}{ Original Values $^{a}$} \\
\hline L. major & 81 & 77 & 4 & 70 & 11 & 73 & 8 \\
\hline heterologous & 24 & 5 & 19 & 6 & 18 & 6 & 18 \\
\hline L. tropica & 15 & 1 & 14 & 2 & 13 & 4 & 11 \\
\hline \multicolumn{8}{|c|}{ Post-TG-ROC Analysis ${ }^{b}$} \\
\hline L. major & 81 & 76 & 5 & 65 & 16 & 70 & 11 \\
\hline heterologous & 24 & 4 & 20 & 7 & 17 & 0 & 24 \\
\hline L. tropica & 15 & 0 & 15 & 3 & 12 & 1 & 14 \\
\hline
\end{tabular}

${ }^{a}$ Values calculated based on the initial cutoff value $\left(C_{\mathrm{i}}\right.$; titer $\left.=1.000\right)$ (Figure 2a), as described in the Supporting Information ("Chemiluminescent ELISA"). ${ }^{b}$ Values calculated based on the TG-ROC analysis (Figure 2c; Tables S1 and S2).

Table 2. Sensitivity, Specificity, and Other Diagnostic Parameters of NGP27b, NGP28b, and NGP30b

\begin{tabular}{|c|c|c|c|c|c|c|}
\hline & \multicolumn{3}{|c|}{ L. major infections vs heterologous diseases } & \multicolumn{3}{|c|}{ L. major vs $L$. tropica infections } \\
\hline & NG27b (\%) & NGP28b (\%) & NGP30b (\%) & NG27b (\%) & NGP28b (\%) & NGP30b (\%) \\
\hline \multicolumn{7}{|c|}{ Original Values $^{a}$} \\
\hline sensitivity $^{b}$ & 95.3 & 88.0 & 91.0 & 95.3 & 88.0 & 91.0 \\
\hline specificity $^{c}$ & 82.8 & 80.0 & 80.0 & 93.8 & 88.2 & 79.0 \\
\hline $\mathrm{FPR}^{d}$ & 17.2 & 20.0 & 20.0 & 6.2 & 11.8 & 21.0 \\
\hline $\mathrm{PPV}^{e}$ & 94.2 & 93.1 & 93.1 & 98.8 & 97.6 & 95.3 \\
\hline $\mathrm{NPV}^{f}$ & 85.7 & 68.6 & 75.0 & 79.0 & 57.7 & 65.2 \\
\hline \multicolumn{7}{|c|}{ Post-TG-ROC Analysis ${ }^{g}$} \\
\hline sensitivity & 94.2 & 83.5 & 88.0 & 95.3 & 88.0 & 88.0 \\
\hline specificity & 85.7 & 92.3 & 100.0 & 100.0 & 88.2 & 93.8 \\
\hline FPR & 14.3 & 7.7 & 0.0 & 0.0 & 11.8 & 6.2 \\
\hline PPV & 95.3 & 92.0 & 100.0 & 100.0 & 96.4 & 98.8 \\
\hline NPV & 82.8 & 60.0 & 75.0 & 75.0 & 48.4 & 57.7 \\
\hline
\end{tabular}

${ }^{a}$ Values calculated based on the initial cutoff value $\left(C_{\mathrm{i}}\right.$; titer $\left.=1.000\right)$ (Figure 2a), as described in the Supporting Information ("Chemiluminescent ELISA"). ${ }^{b}$ Sensitivity $=$ true positive $(\mathrm{TP}) / \mathrm{TP}+$ false negative $(\mathrm{FN}) .{ }^{c}$ Specificity $=$ true negative $(\mathrm{TN}) / \mathrm{TN}+$ false positive $(\mathrm{FP}) .{ }^{d}$ False-positive rate $=100-$ specificity. ${ }^{e}$ Positive predictive value $=\mathrm{TP} / \mathrm{TP}+\mathrm{FP} .{ }^{f}$ Negative predictive value $=\mathrm{TN} / \mathrm{TN}+\mathrm{FN} .{ }^{g}$ Values calculated based on the TGROC analysis (Figure 2c; Tables S1 and S2).

Second, we evaluated these NGPs for their utility for distinguishing $L$. major from $L$. tropica infections, which is another challenge in similar clinical settings. To that end, we assessed the three NGPs by chemiluminescent ELISA, using conditions previously established (Figure S5). We assayed individual sera from CL patients chronically infected with $L$. major $(n=81)$ or L. tropica $(n=15)$ or patients with heterologous diseases $(n=24)$ (Table S1). We initially used a chemiluminescent ELISA titer cutoff of 1.000, which was determined in each immunoassay microplate by using a pool of negative control sera (healthy individuals from the UK, $n=$ 10), in duplicate or triplicate, as described in detail in the Supporting Information ("Chemiluminescent ELISA") (Figure 2A and Figure S6a). Our data showed that NGP27b diagnosed as positive $77 / 81$ (sensitivity $=95.3 \%$ ) of the sera from patients with $L$. major infection, previously confirmed by dermatological examination and laboratory assays (lesion aspirate microscopy and ITS1-PCR-RFLP analysis) ${ }^{63}$ (Supporting Information, "Cohort description") (Figure 2a; Tables 1 and 2). On the other hand, NGP28b and NGP30b diagnosed as L. major-positive $70 / 81$ and $73 / 81$ (sensitivity $=88.0 \%$ and $91.0 \%$ ), respectively. We also evaluated the three NGPs for specificity by comparing sera from $L$. major infections with sera from heterologous diseases or $L$. tropica infections. When we assessed L. major-positive sera vs sera from heterologous diseases, NGP27b, NGP28b, and NGP30b exhibited a specificity of $82.8 \%, 80.0 \%$, and $80.0 \%$, respectively (Table 2). When we compared L. major-positive vs $L$. tropicapositive sera, we found that NGP27b, NGP28b, and NGP30b showed a specificity of $93.8 \%, 88.2 \%$, and $79.0 \%$, respectively (Table 2). Conversely, individual or pooled sera from L. major or L. tropica infections or heterologous diseases showed very weak reactivity (mostly below the titer cutoff of 1.000) with the negative control antigen (2-MEb), strongly indicating that the antibody reactivity of all tested sera to the linker or the BSA carrier protein was negligible (Figure S6b).

To compare the usefulness of the three NGPs for correctly discriminating true-positive (TP) from false-positive (FP) results, at various threshold (cutoff) values, we plotted receiver-operating characteristic (ROC) curves (Figure 2b). The area under the curve (AUC) values of the ROC curves for NGP27b (0.9421), NGP28b (0.9216), and NGP30b (0.9159), in the comparison of serum samples from L. major infections vs heterologous diseases, indicated that NGP27b exhibited higher sensitivity and specificity than NGP28b and NGP30b (Figure 2b, top graphs; Table 2). In the comparison of serum samples from $L$. major vs $L$. tropica infections, the AUC values for NGP27b (0.9757), NGP28b (0.9280), and NGP30b (0.8951) indicated the same trend (Figure 2b, bottom graphs; Table 2). Taken together, our initial data indicated that NGP27b showed a higher sensitivity and specificity than NGP28b and NGP30b. 
Next, to fine-tune the initial titer cutoff value $\left(C_{\mathrm{i}}\right.$; Figure 2a) for each NGP, we performed a two-graph ROC (TG-ROC) analysis by plotting the ROC data (Figure $2 \mathrm{~b}$ ) for sensitivity $(\mathrm{Se})$ and specificity $(\mathrm{Sp})$ as a function of the cutoff value, as described by Greiner et al. ${ }^{64}$ (Figure $2 \mathrm{c}$ ). The selection of the cutoff value is always a trade-off between sensitivity and specificity, and it depends on the context in which the diagnostic BMK is to be applied. Since CL is caused by $L$. major or L. tropica in endemic countries like Saudi Arabia, where little geographical overlap of the two infections exists, the utility of a new diagnostic BMK test is not a high priority. However, in areas where L. major and L. tropica infections coexist, for instance, in conflict-affected countries (e.g., Afghanistan, Syria, Lebanon $)^{65,66}$ and nonendemic regions (e.g., Europe), with high migration from affected areas, ${ }^{67,68}$ there is an urgent need for new diagnostic BMKs that could accurately diagnose CL from non-CL conditions (i.e., dermatological diseases) and discriminate CL caused by different Leishmania species. In this context, a high specificity is preferred over high sensitivity for any potential new diagnostic BMK for CL. ${ }^{64}$ When comparing L. major infections vs heterologous diseases, an adjusted titer cutoff value for NGP27b of 1.140 (instead of 1.000) slightly decreased sensitivity to $94.2 \%$ (from $95.3 \%$ ) but increased specificity to 85.7\% (from $82.8 \%$ ) (Table 2). When comparing L. major vs L. tropica infections, we noticed that an adjusted titer cutoff value for NGP27b of 1.045 afforded a perfect specificity of $100 \%$ (from 93.8\%), while maintaining the same high sensitivity of $95.3 \%$ (Figure 2a,c; Table 2). For NGP28b, in the comparison of $L$. major infections vs heterologous diseases, an adjusted titer cutoff value of 1.600 gave a lower sensitivity of $83.5 \%$ (from $88.0 \%$ ), while the specificity considerably improved to $92.3 \%$ (from $80.0 \%$ ). Nevertheless, when comparing $L$. major vs $L$. tropica infections, we found that the original NGP28b titer cutoff value of 1.000 could not be significantly improved without drastically affecting the sensitivity. Therefore, we maintained the same cutoff value of 1.000 (Figure 2a,c; Table 2). Finally, for NGP30b, when we compared L. major infections vs heterologous diseases, an adjusted titer cutoff value of 1.465 gave a sensitivity of $88 \%$ (from 91\%) but significantly increased the specificity to $100 \%$ (from $80.0 \%$ ) (Figure 2a,c; Table 2). When comparing $L$. major vs $L$. tropica infections with NGP30b, the same adjusted titer cutoff value of 1.465 decreased the sensitivity to $88 \%$ (from $91.0 \%$ ) but significantly increased the specificity to 93.8\% (from $79.0 \%$ ).

Based on the TG-ROC analysis data with the adjusted titer cutoff values, we can propose an algorithm with the two NGPs that exhibited the best outcomes in terms of sensitivity and specificity, i.e., NGP27b and NGP30b, to consecutively screen sera from patients who could be infected with either L. major or L. tropica, or affected with a confounding, non-CL dermatological condition(s) (Figure 3). First, the serum would be screened by chemiluminescent ELISA with NGP27b. A positive result would indicate L. major infection or heterologous disease, whereas a negative result would indicate L. tropica infection or heterologous disease. A sample with a positive result would undergo a second chemiluminescent ELISA now using NGP30b to discriminate between $L$. major infection and heterologous disease. Importantly, the proposed algorithm should complement the patient's clinical assessment and history.

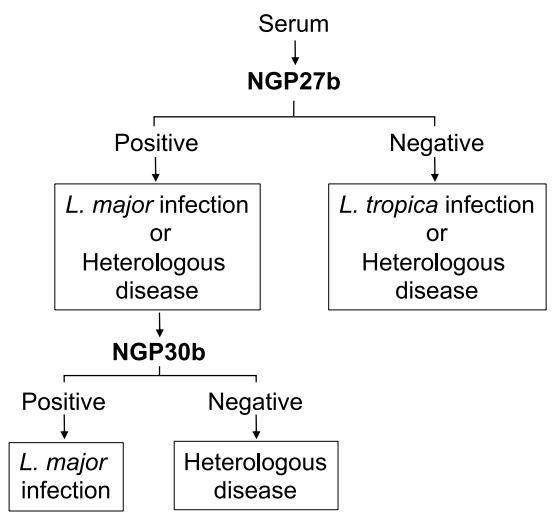

Figure 3. Algorithm for discriminating L. major infection from $L$. tropica infection and heterologous disease, using NGP27b and NGP30b sequentially in chemiluminescent ELISA.

Although all three NGPs exhibited similar trends regarding their immunoreactivity with $L$. major sera, on average, NGP30b showed titers about twice as high as those of NGP27b and NGP28b (Figure 2a). This could be explained by the fact that NGP30b contains a larger portion of the glycotope of type-2 GIPL-2, which has been shown to be strongly recognized by sera from $L$. major patients. ${ }^{38}$

Regardless of the NGP tested, we observed a small crossreactivity with sera from $L$. tropica infections and heterologous diseases. A plausible explanation for such a cross-reactivity could be the presence of natural anti- $\alpha$-Gal antibodies, abundantly present in the serum of all individuals, as reported by Galili et al. ${ }^{61,62}$ These antibodies cross-react with the socalled Galili epitope or trisaccharide (Gal $\alpha 1,3 \mathrm{Gal} \beta 1,4 \mathrm{Glc}$ $\mathrm{NAc} \beta$ ) and other glycans containing terminal, nonreducing $\alpha$ Gal epitopes, in particular melibiose (Gal $\alpha 1,6 \mathrm{Glc}) .{ }^{12,18,46,52,53,69}$ In fact, both the Galili trisaccharide and melibiose are regularly used for the purification of natural anti- $\alpha$-Gal antibodies, ${ }^{12,70}$ which could explain at least in part the cross-reactivity observed here with NGP27b (Gal $\alpha 1,3 \mathrm{Gal} f \beta$-BSA) and NGP30b (Gal $\alpha 1,3 \mathrm{Gal} f \beta 1,3 \mathrm{Man} \alpha$ BSA) and NGP28b (Gal $\alpha 1,6 \mathrm{Gal} \alpha 1,3 \mathrm{Gal} f \beta$-BSA), which contain terminal $\alpha$-Gal residues with the same linkages as observed in the Galili trisaccharide and melibiose.

Similar bottom-up reversed immunoglycomics studies for the discovery of glycotopes and BMKs have been previously described in the trematode Schistosoma mansoni and kinetoplastids closely related to $L$. major, i.e., T. cruzi and $L$. braziliensis. For instance, Naus and colleagues used surface plasmon resonance (SPR) to evaluate three NGPs containing synthetic S. mansoni-derived glycans, i.e., FLDN [Fuc $\alpha 1-$ $3 \mathrm{Gal} p \mathrm{NAc} \beta 1-4 \mathrm{Glc} p \mathrm{NAc} \beta 1-3 \mathrm{Gal} p \alpha 1]$, LDN-DF (GalpNAc $\beta 1-4[\operatorname{Fuc} \alpha 1-2 \mathrm{Fuc} \alpha 1-3]$ Glc $p$ NAc $\beta 1-3 \mathrm{Gal} p \alpha 1)$, and LDNF (Gal $p N A c \beta 1-4(\operatorname{Fuc} \alpha 1-3)$ Glc $p N A c \beta 1-3 G a l p \alpha 1)$, with two cohorts $(n=235)$ from an endemic area in Kenya. $^{71}$ They found that LDN-DF was the most antigenic NGP, being recognized especially by young children with early infection. In a subsequent study, the same group of investigators and collaborators, using monoclonal antibodies (mAbs), raised against the immunogenic $O$-glycan of the $S$. mansoni circulating anodic antigen (CAA) repeating unit $([\rightarrow$ $6)$ - $[\beta$-D-Glc $p \mathrm{~A}-(1 \rightarrow 3)]-\beta$-D-GalpNAc- $\left.(1 \rightarrow]_{n}\right)$ (anti-CAA mAbs), showed that this disaccharide was strongly recognized by urine and serum from patients with schistosomiasis. ${ }^{72}$ To get insight into the specificity of these anti-CAA mAbs, a series 
of synthetic di-, tri-, and tetrasaccharide analogues of the CAA repeating unit were evaluated as NGPs by sandwich ELISA and SPR. ${ }^{73}$ Those authors showed that these mAbs were more reactive to the repeating unit dimer than to the monomer, indicating a potential application of these mAbs as diagnostic tools to detect circulating $S$. mansoni antigens.

In a more recent study on CL caused by New-World $L$. braziliensis, de Souza and colleagues analyzed the serum immunoreactivity to three commercial NGPs (non-Leishmania-derived) containing terminal, nonreducing Gal $p \alpha 1,3 \mathrm{Gal} p \beta$ based glycotopes by chemiluminescent ELISA, using sera from (i) L. braziliensis-caused CL patients, all with active lesion, subjected or not to treatment, or undergoing treatment; (ii) healthy individuals from endemic and nonendemic areas in Brazil; and (iii) individuals with heterologous diseases (CD, hepatitis B or C, tuberculosis, or syphilis). ${ }^{74}$ Interestingly, they found that the highest sensitivity and specificity were observed to the trisaccharide Galp $\alpha 1,3 \mathrm{Gal} p \beta 1,4 \mathrm{Glc} p \mathrm{NAc} \beta$ (Galili's trisaccharide), which has not yet been described in $L$. braziliensis and is known to react very strongly with anti- $\alpha$ Gal Abs from CD patients. ${ }^{52,53,69}$ We had also previously observed very high levels of anti- $\alpha$-Gal Abs to the same NGP containing the Galili's trisaccharide in the serum of patients with Old-World CL caused by L. major or L. tropica. ${ }^{45}$ Although we observed a significant difference in the anti- $\alpha$-Gal $\mathrm{Ab}$ titers between sera of L. major and L. tropica infections, no differential diagnosis between these two infections was achieved using the Gal $p \alpha 1,3 \mathrm{Gal} p \beta 1,4 \mathrm{Gl} c p \mathrm{NAc}$ epitope. In a more recent study, we assayed several other NGPs containing other $\alpha$-Gal-glycotopes linked to BSA, including $\alpha$-Gal $p$ (NGP3b), Galp $\alpha 1,3 \mathrm{Gal} p \alpha$ (NGP17b), Galp $\alpha 1,3 \mathrm{Gal} p \beta$ (NGP9b), Galp $\alpha 1,6[\mathrm{Gal} p \alpha 1,2] \mathrm{Gal} p \beta$ (NGP11b), and Gal$p \alpha 1,3 \mathrm{Gal} p \beta 1,4 \mathrm{Glc} p \beta$ (NGP1b). ${ }^{46}$ When assayed by chemiluminescent ELISA with sera from L. major-infected patients with active CL lesion $(n=17)$ or cured $(n=29)$ or sera from heterologous diseases, the most promising diagnostic potential $\left(\mathrm{AUC}_{\mathrm{ROC}}=0.8\right)$ was observed with NGP3b $(\alpha$-Galp-BSA $)$. Although this AUC value is considered acceptable for a diagnostic test, a higher accuracy (AUC $=0.9-1.0$ ), as we observed in most ROC curves in this study, is desirable. ${ }^{75,76}$ Moreover, we and others have also been developing a similar reverse immunoglycomics approach for the development of diagnostic and/or prognostic tools (for early assessment of chemotherapeutic outcomes) in CD, using NGPs displaying synthetic terminal, nonreducing $\alpha$-Galp glycotopes derived from $T$. cruzi or recognized by patients with chronic CD. ${ }^{52,53,69,77,78}$ These studies have shown very promising results with highly immunogenic synthetic $\alpha$-Gal glycotopes as BMKs for diagnosis and follow-up of chemotherapy in the context of $\mathrm{CD}$. However, all potential BMKs revealed by the reverse immunoglycomics approach, regardless of the disease, need to be extensively validated using a more comprehensive and larger panel of sera of homologous and heterologous diseases and healthy individuals under strict clinical laboratory and/or field settings.

\section{CONCLUSION}

The chemical synthesis of $\alpha$-Gal-containing oligosaccharides and their immunological evaluation with sera from OWCL patients enabled the identification of diagnostic BMKs for distinguishing OWCL caused by L. major from heterologous diseases and from L. tropica infections. The synthetic targets were the 3-thiopropyl glycosides of Galp $\alpha 1,3 \mathrm{Gal} f \beta\left(\mathbf{G} 27_{\mathrm{SH}}\right)$,
Galp $\alpha 1,3 \mathrm{Gal} f \beta 1,3 \mathrm{Man} \alpha\left(\mathbf{G}_{30} \mathbf{S H}_{\mathrm{SH}}\right)$, and Gal $p \alpha 1,6 \mathrm{Gal} p \alpha 1,3 \mathrm{Gal} f \beta$ $\left(\mathbf{G} 28_{\mathrm{SH}}\right)$ equipped with a handle for conjugation. These oligosaccharides correspond to the terminal di- and trisaccharide moieties of GIPL-2 and the terminal trisaccharide of GIPL3 , respectively, which are abundantly expressed in L. major but absent (or much less abundant) in L. tropica. The 4,6-O-DTBS protecting group of Galp played two important roles in their synthesis: (a) it allowed for stereoselective $\alpha$-galactosylation; ${ }^{50}$ and (b) it underwent a regioselective ring-opening reaction producing a new Gal acceptor in a single step, which allowed for a convenient synthesis of $\mathbf{G} \mathbf{2 8} \mathbf{S H}_{\mathbf{S H}}$. Conjugation of the saccharides $\mathbf{G 2 7} \mathbf{S H}_{\mathrm{SH}}, \mathbf{G} \mathbf{2 8} \mathbf{S}_{\mathrm{SH}}$, and $\mathbf{G} 3 \mathbf{S}_{\mathrm{SH}}$ to maleimidederivatized BSA produced NGP antigens for chemiluminescent ELISA. The two NGPs, NGP27b and NGP30b, both derived from L. major GIPL-2, exhibited $100 \%$ specificity for the distinction of L. major from L. tropica infections (NGP27b) and heterologous diseases (NGP30b). Therefore, sera of patients with skin lesions that are suspicious for OWCL can be subjected to two consecutive chemiluminescent ELISA tests, which will diagnose an L. major infection with a very high level of confidence. These NGPs could potentially be used to develop a species-specific lateral flow test for OWCL, which is important for informing best treatment options. This is especially relevant in areas of population displacement in the Middle East with large numbers of refugees who migrated from OWCL-endemic areas.

The reversed immunoglycomics approach presented here shows that glycotope and diagnostic BMK discovery does not necessarily require cultivation of large amounts of parasite, isolation, and analysis of glycans from the highly diverse and heterogeneous glycocalyx. Instead, one can take advantage of known structural information and probe the antigenicity of small synthetic glycan partial structures in serological assays using patient sera. Taken together, our results suggest that $\alpha$ Gal glycotopes of other closely related kinetoplastid (i.e., $T$. cruzi) and New-World Leishmania species (e.g., L. braziliensis and L. mexicana $)^{10,23,39}$ and as-yet unidentified microbiomederived $\alpha$-Gal glycotopes that elicit anti- $\alpha$-Gal Abs present in healthy individuals could potentially be unraveled in a similar manner.

\section{ASSOCIATED CONTENT}

\section{Supporting Information}

The Supporting Information is available free of charge at https://pubs.acs.org/doi/10.1021/jacsau.1c00201.

Detailed experimental procedures of carbohydrate syntheses and compound characterization; synthesis of NGPs; synthesis of $2-\mathrm{MEb}$ (negative control); ethics statement and cohort description; description of the chemiluminescent ELISA; cross-titrations of NGP antigens and $2-\mathrm{MEb}$ with pooled sera of OWCL patients as well as healthy individuals by chemiluminescent ELISA; chemiluminescent ELISA reactivity of NGPs and $2-\mathrm{MEb}$ with individual sera from OWCL patients, heterologous disease, or from healthy individuals; ${ }^{1} \mathrm{H}$ and ${ }^{13} \mathrm{C}$ NMR spectra; and MALDI-TOF and ESI-TOF mass spectra (PDF)

\section{AUTHOR INFORMATION}

\section{Corresponding Authors}

Álvaro Acosta-Serrano - Department of Vector Biology, Department of Tropical Disease Biology, Liverpool School of 
Tropical Medicine, Liverpool L3 5QA, United Kingdom; Email: alvaro.acosta-serrano@lstmed.ac.uk

Igor C. Almeida - Department of Biological Sciences, Border Biomedical Research Center, University of Texas at El Paso, El Paso, Texas 79968, United States; Email: icalmeida@ utep.edu

Katja Michael - Department of Chemistry and Biochemistry, Border Biomedical Research Center, University of Texas at El Paso, El Paso, Texas 79968, United States; 10 orcid.org/ 0000-0001-6754-3702; Email: kmichael@utep.edu

\section{Authors}

Alba L. Montoya - Department of Chemistry and Biochemistry, Border Biomedical Research Center, University of Texas at El Paso, El Paso, Texas 79968, United States; Present Address: Department of Medicinal Chemistry, University of Utah, 30 South 2000 East, Salt Lake City, UT 84112, USA

Victoria M. Austin - Department of Vector Biology, Department of Tropical Disease Biology, Liverpool School of Tropical Medicine, Liverpool L3 SQA, United Kingdom; Present Address: Ninewells Hospital and Medical School, Dundee DD2 1SG, UK; 다이.org/0000-0001-98628610

Susana Portillo - Department of Biological Sciences, Border Biomedical Research Center, University of Texas at El Paso, El Paso, Texas 79968, United States; Present Address: University of Maryland School of Medicine, Center for Vaccine Development and Global Health, 685 West Baltimore St., Baltimore, MD 21201, USA

Irodiel Vinales - Department of Chemistry and Biochemistry, Border Biomedical Research Center, University of Texas at El Paso, El Paso, Texas 79968, United States

Roger A. Ashmus - Department of Chemistry and Biochemistry, Border Biomedical Research Center, University of Texas at El Paso, El Paso, Texas 79968, United States; Present Address: Department of Chemistry, Simon Fraser University, 8888 University Drive, Burnaby, BC V5A 1S6, Canada

Igor Estevao - Department of Biological Sciences, Border Biomedical Research Center, University of Texas at El Paso, El Paso, Texas 79968, United States; ㅇo․ orid.org/00000003-3946-8375

Sohan R. Jankuru - Department of Chemistry and Biochemistry, Border Biomedical Research Center, University of Texas at El Paso, El Paso, Texas 79968, United States

Yasser Alraey - Department of Vector Biology, Department of Tropical Disease Biology, Liverpool School of Tropical Medicine, Liverpool L3 5QA, United Kingdom; Present Address: Department of Clinical Laboratory Sciences, College of Applied Medical Sciences, King Khalid University, Abha, Saudi Arabia

Waleed S. Al-Salem - Department of Vector Biology, Department of Tropical Disease Biology, Liverpool School of Tropical Medicine, Liverpool L3 5QA, United Kingdom; Present Address: Zarga Alyamamah Street, Al Murabba, Riyadh 12628, Saudi Arabia

Complete contact information is available at: https://pubs.acs.org/10.1021/jacsau.1c00201

\section{Author Contributions}

${ }$ A.L.M. and V.M.A. contributed equally to this work.

\section{Author Contributions}

A.L.M. synthesized and characterized the oligosaccharides and neoglycoproteins and wrote parts of the manuscript. V.M.A. performed chemiluminescent ELISA. S.P. performed chemiluminescent ELISA and characterized neoglycoproteins. I.V. synthesized and characterized oligosaccharides. R.A.A. discovered the regioselective opening of the 4,6-O-DTBS ring on Galp. I.E. organized and analyzed ELISA data. S.R.J. performed NMR analyses. Y.A. and W.S.A.-S. collected and processed sera and confirmed L. major or L. tropica infection by PCR. A.A.-S. supervised the sera collection and the individual ELISA, analyzed data, and proofread the manuscript. I.C.A. supervised the ELISA using pooled sera and the MALDI-TOF analysis, performed the receiver-operating characteristic analysis, and wrote parts of the manuscript. K.M. supervised the synthesis and characterization of the oligosaccharides, performed purifications by FPLC, and wrote parts of the manuscript.

Notes

The authors declare no competing financial interest.

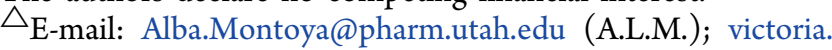
austin@nhs.scot (V.M.A.); sportillo@som.umaryland.edu (S.P.); roger_ashmus@sfu.ca (R.A.A.); yahamd@kku.edu.sa (Y.A.); walsalem@moh.gov.sa (W.S.A.-S.).

\section{ACKNOWLEDGMENTS}

This work was supported by NIH grant 1R21AI137890-01 (K.M. and A.A.-S.), a Dodson research grant from the University of Texas at El Paso (S.P.), and an NPT-UK grant (A.A.-S.). A.L.M. and S.P. are grateful for a Dr. Keelung Hong Graduate Research Fellowship. W.S.A.-S. and Y.A. were supported with a $\mathrm{PhD}$ studentship from the Saudi Cultural Bureau. The authors are thankful to the Biomolecule Analysis and Omics Unit (BAOU), at BBRC/UTEP, supported by the grant 2G12MD007592 (to Robert A. Kirken), from the National Institute on Minority Health and Health Disparities (NIMHD), for the full access to the MALDI-TOF-MS and other core instruments used in this study.

\section{REFERENCES}

(1) Galili, U.; Shohet, S. B.; Kobrin, E.; Stults, C. L.; Macher, B. A. Man, apes, and Old World monkeys differ from other mammals in the expression of alpha-galactosyl epitopes on nucleated cells. J. Biol. Chem. 1988, 263, 17755-17762.

(2) Galili, U.; Mandrell, R. E.; Hamadeh, R. M.; Shohet, S. B.; Griffis, J. M. Interaction between human natural anti- $\alpha$-galactosyl immunoglobulin G and bacteria of the human flora. Infect. Immun. 1988, 56, 1730-1737.

(3) Galili, U.; Rachmilewitz, E. A.; Peleg, A.; Flechner, I. A Unique Natural Human IgG Antibody With Anti-Alpha-Galactosyl Specificity. J. Exp. Med. 1984, 160 (5), 1519-1531.

(4) Galili, U. Interaction of the natural anti-Gal antibody with alphagalactosyl epitopes: a major obstacle for xenotransplantation in humans. Immunol. Today 1993, 14, 480-482.

(5) Galili, U. The Natural Anti-Gal Antibody As Foe Turned Friend In Medicine, 1st ed.; Academic Press, Elsevier: London, 2018, pp. 171-228.

(6) Carlson, C. B.; Mowery, P.; Owen, R. M.; Dykhuizen, E. C.; Kiessling, L. L. Selective Tumor Cell Targeting Using Low-Affinity, Multivalent Interactions. ACS Chem. Biol. 2007, 2 (2), 119-127.

(7) Sianturi, J.; Manabe, Y.; Li, H.-S.; Chiu, L.-T.; Chang, T.-C.; Tokunaga, K.; Kabayama, K.; Tanemura, M.; Takamatsu, S.; Miyoshi, E.; Hung, S.-C.; Fukase, K. Development of $\alpha$-Gal-Antibody Conjugates to Increase Immune Response by Recruiting Natural Antibodies. Angew. Chem., Int. Ed. 2019, 58 (14), 4526-4530. 
(8) LaTemple, D. C.; Abrams, J. T.; Zhang, S. Y.; Galili, U. Increased immunogenicity of tumor vaccines complexed with anti-Gal: studies in knockout mice for $\alpha 1$,3galactosyltranferase. Cancer Res. 1999, 56, 3069-3074.

(9) Galili, U. Amplifying immunogenicity of prospective Covid-19 vaccines by glycoengineering the coronavirus glycan-shield to present alpha-gal epitopes. Vaccine 2020, 38, 6487-6499.

(10) Avila, J. L.; Rojas, M.; Galili, U. Immunogenic Gal alpha 1-— $3 \mathrm{Gal}$ carbohydrate epitopes are present on pathogenic American Trypanosoma and Leishmania. J. Immunol. 1989, 142 (8), 28282834.

(11) Avila, J. L. alpha-Galactosyl-bearing epitopes as potent immunogens in Chagas' disease and leishmaniasis. Subcell. Biochem. 1999, 32, 173-213.

(12) Almeida, I. C.; Milani, S. R.; Gorin, P. A.; Travassos, L. R. Complement-mediated lysis of Trypanosoma cruzi trypomastigotes by human anti-alpha-galactosyl antibodies. J. Immunol. 1991, 146 (7), 2394-2400.

(13) Milani, S. R.; Travassos, L. R. Anti-alpha-galactosyl antibodies in chagasic patients. Possible biological significance. Braz. J. Med. Biol. Res. 1988, 21 (6), 1275-1286.

(14) Ramasamy, R.; Field, M. C. Terminal galactosylation of glycoconjugates in Plasmodium falciparum asexual blood stages and Trypanosoma brucei bloodstream trypomastigotes. Exp. Parasitol. 2012, 130 (4), 314-320.

(15) Aguilar, R.; Ubillos, I.; Vidal, M.; Balanza, N.; Crespo, N.; Jimenez, A.; Nhabomba, A.; Jairoce, C.; Dosoo, D.; Gyan, B.; Ayestaran, A.; Sanz, H.; Campo, J. J.; Gomez-Perez, G. P.; Izquierdo, L.; Dobano, C. Antibody responses to alpha-Gal in African children vary with age and site and are associated with malaria protection. Sci. Rep. 2018, 8 (1), 9999.

(16) Ravindran, B.; Satapathy, A. K.; Das, M. K. Naturally-occurring anti-alpha-galactosyl antibodies in human Plasmodium falciparum infections-a possible role for autoantibodies in malaria. Immunol. Lett. 1988, 19 (2), 137-141.

(17) Ramasamy, R.; Reese, R. T. Terminal galactose residues and the antigenicity of Plasmodium falciparum glycoproteins. Mol. Biochem. Parasitol. 1986, 19 (2), 91-101.

(18) Almeida, I. C.; Ferguson, M. A.; Schenkman, S.; Travassos, L. R. Lytic anti-alpha-galactosyl antibodies from patients with chronic Chagas' disease recognize novel O-linked oligosaccharides on mucinlike glycosyl-phosphatidylinositol-anchored glycoproteins of Trypanosoma cruzi. Biochem. J. 1994, 304 (3), 793-802.

(19) Chernykh, A.; Kawahara, R.; Thaysen-Andersen, M. Towards structure-focused glycoproteomics. Biochem. Soc. Trans. 2021, 49 (1), $161-186$.

(20) Wilkinson, H.; Saldova, R. Current Methods for the Characterization of O-Glycans. J. Proteome Res. 2020, 19 (10), 3890-3905.

(21) Borza, B.; Hajba, L.; Guttman, A. N-glycan Analysis in Molecular Medicine: Innovator and Biosimilar Protein Therapeutics. Curr. Mol. Med. 2021, 20 (10), 828-839.

(22) Rodrigues, J. A.; Acosta-Serrano, A.; Aebi, M.; Ferguson, M. A.; Routier, F. H.; Schiller, I.; Soares, S.; Spencer, D.; Titz, A.; Wilson, I. B.; Izquierdo, L. Parasite Glycobiology: A Bittersweet Symphony. PLoS Pathog. 2015, 11 (11), e1005169.

(23) McConville, M. J.; Ferguson, M. A. The structure, biosynthesis and function of glycosylated phosphatidylinositols in the parasitic protozoa and higher eukaryotes. Biochem. J. 1993, 294 (2), 305-324.

(24) de Vries, H. J.; Reedijk, S. H.; Schallig, H. D. Cutaneous leishmaniasis: recent developments in diagnosis and management. Am. J. Clin. Dermatol. 2015, 16 (2), 99-109.

(25) Alvar, J.; Vélez, I. D.; Bern, C.; Herrero, M.; Desjeux, P.; Cano, J.; Jannin, J.; den Boer, M.; Team, W. L. C. Leishmaniasis worldwide and global estimates of its incidence. PLoS One 2012, 7 (5), e35671.

(26) Bailey, F.; Mondragon-Shem, K.; Haines, L. R.; Olabi, A.; Alorfi, A.; Ruiz-Postigo, J. A.; Alvar, J.; Hotez, P.; Adams, E. R.; Vélez, I. D.; Al-Salem, W.; Eaton, J.; Acosta-Serrano, Á.; Molyneux, D. H. Cutaneous leishmaniasis and co-morbid major depressive disorder: A systematic review with burden estimates. PLoS Neglected Trop. Dis. 2019, 13 (2), e0007092.

(27) Masmoudi, A.; Hariz, W.; Marrekchi, S.; Amouri, M.; Turki, H. Old World cutaneous leishmaniasis: diagnosis and treatment. J. Dermatol. Case Rep. 2013, 7 (2), 31-41.

(28) Handler, M. Z.; Patel, P. A.; Kapila, R.; Al-Qubati, Y.; Schwartz, R. A. Cutaneous and mucocutaneous leishmaniasis: Differential diagnosis, diagnosis, histopathology, and management. J. Am. Acad. Dermatol. 2015, 73 (6), 911-926.

(29) Al-Salem, W. S.; Solórzano, C.; Weedall, G. D.; Dyer, N. A.; Kelly-Hope, L.; Casas-Sánchez, A.; Alraey, Y.; Alyamani, E. J.; Halliday, A.; Balghonaim, S. M.; Alsohibany, K. S.; Alzeyadi, Z.; Alzahrani, M. H.; Al-Shahrani, A. M.; Assiri, A. M.; Memish, Z.; Acosta-Serrano, A. Old World cutaneous leishmaniasis treatment response varies depending on parasite species, geographical location and development of secondary infection. Parasites Vectors 2019, 12 (1), 195.

(30) Saab, J.; Fedda, F.; Khattab, R.; Yahya, L.; Loya, A.; Satti, M.; Kibbi, A. G.; Houreih, M. A.; Raslan, W.; El-Sabban, M.; Khalifeh, I. Cutaneous leishmaniasis mimicking inflammatory and neoplastic processes: a clinical, histopathological and molecular study of 57 cases. J. Cutaneous Pathol. 2012, 39 (2), 251-262.

(31) Masmoudi, A.; Hariz, W.; Marrekchi, S.; Amouri, M.; Turki, H. Old World cutaneous leishmaniasis: diagnosis and treatment. J. Dermatol. Case Rep. 2013, 7 (2), 31-41.

(32) Du, R.; Hotez, P. J.; Al-Salem, W. S.; Acosta-Serrano, A. Old World Cutaneous Leishmaniasis and Refugee Crises in the Middle East and North Africa. PLoS Neglected Trop. Dis. 2016, 10 (5), e0004545.

(33) Cabezas, Y.; Legentil, L.; Robert-Gangneux, F.; Daligault, F.; Belaz, S.; Nugier-Chauvin, C.; Tranchimand, S.; Tellier, C.; Gangneux, J. P.; Ferrières, V. Leishmania cell wall as a potent target for antiparasitic drugs. A focus on the glycoconjugates. Org. Biomol. Chem. 2015, 13 (31), 8393-8404.

(34) Schneider, P.; Schnur, L. F.; Jaffe, C. L.; Ferguson, M. A.; McConville, M. J. Glycoinositol-phospholipid profiles of four serotypically distinct Old World Leishmania strains. Biochem. J. 1994, 304 (2), 603-609.

(35) Forestier, C. L.; Gao, Q.; Boons, G. J. Leishmania lipophosphoglycan: how to establish structure-activity relationships for this highly complex and multifunctional glycoconjugate? Front. Cell. Infect. Microbiol. 2015, 4, 193.

(36) Guha-Niyogi, A.; Sullivan, D. R.; Turco, S. J. Glycoconjugate structures of parasitic protozoa. Glycobiology 2001, 11 (4), 45R-59R.

(37) McConville, M. J.; Bacic, A. A family of glycoinositol phospholipids from Leishmania major. Isolation, characterization, and antigenicity. J. Biol. Chem. 1989, 264 (2), 757-766.

(38) McConville, M. J.; Homans, S. W.; Thomas-Oates, J. E.; Dell, A.; Bacic, A. Structures of the glycoinositolphospholipids from Leishmania major. A family of novel galactofuranose-containing glycolipids. J. Biol. Chem. 1990, 265 (13), 7385-7394.

(39) Avila, J. L.; Rojas, M.; Acosta, A. Glycoinositol phospholipids from American Leishmania and Trypanosoma spp: partial characterization of the glycan cores and the human humoral immune response to them. J. Clin. Microbiol. 1991, 29 (10), 2305-2312.

(40) Rosen, G.; Londner, M. V.; Sevlever, D.; Greenblatt, C. L. Leishmania major: glycolipid antigens recognized by immune human sera. Mol. Biochem. Parasitol. 1988, 27 (1), 93-99.

(41) Schnaidman, B. B.; Yoshida, N.; Gorin, P. A.; Travassos, L. R. Cross-reactive polysaccharides from Trypanosoma cruzi and fungi (especially Dactylium dendroides). J. Protozool. 1986, 33 (2), 186191.

(42) Travassos, L. R.; Almeida, I. C. Carbohydrate immunity in American trypanosomiasis. Springer Semin. Immunopathol. 1993, 15 (2-3), 183-204.

(43) Galili, U. Anti-Gal in Humans and Its Antigen the $\alpha$-Gal Epitope. In The Natural Anti-Gal Antibody As Foe Turned Friend In Medicine, 1st ed.; Galili, U., Ed.; Academic Press, Elsevier: London, 2018; pp 1-18. 
(44) Wilkinson, S. G. Bacterial lipopolysaccharides-themes and variations. Prog. Lipid Res. 1996, 35, 283-343.

(45) Al-Salem, W. S.; Ferreira, D. M.; Dyer, N. A.; Alyamani, E. J.; Balghonaim, S. M.; Al-Mehna, A. Y.; Al-Zubiany, S.; Ibrahim, e.-K.; Al Shahrani, A. M.; Alkhuailed, H.; Aldahan, M. A.; Al Jarallh, A. M.; Abdelhady, S. S.; Al-Zahrani, M. H.; Almeida, I. C.; Acosta-Serrano, A. Detection of high levels of anti- $\alpha$-galactosyl antibodies in sera of patients with Old World cutaneous leishmaniasis: a possible tool for diagnosis and biomarker for cure in an elimination setting. Parasitology 2014, 141 (14), 1898-1903.

(46) Subramaniam, K. S.; Austin, V.; Schocker, N. S.; Montoya, A. L.; Anderson, M. S.; Ashmus, R. A.; Mesri, M.; Al-Salem, W.; Almeida, I. C.; Michael, K.; Acosta-Serrano, A. Anti- $\alpha$-Gal antibodies detected by novel neoglycoproteins as a diagnostic tool for Old World cutaneous leishmaniasis caused by Leishmania major. Parasitology 2018, 145 (13), 1758-1764.

(47) Ruda, K.; Lindberg, J.; Garegg, P. J.; Oscarson, S.; Konradsson, P. Synthesis of the Leishmania LPG Core Heptasaccharyl myoInositol. J. Am. Chem. Soc. 2000, 122 (45), 11067-11072.

(48) Gandolfi-Donadio, L.; Gallo-Rodriguez, C.; de Lederkremer, R. M. Synthesis of alpha-D-Galp-(1->3)-beta-D-Galf-(1->3)-D-man, a terminal trisaccharide of Leishmania type-2 glycoinositolphospholipids. J. Org. Chem. 2002, 67 (13), 4430-4435.

(49) Almeida, I. C.; Covas, D. T.; Soussumi, L. M.; Travassos, L. R. A highly sensitive and specific chemiluminescent enzyme-linked immunosorbent assay for diagnosis of active Trypanosoma cruzi infection. Transfusion 1997, 37 (8), 850-857.

(50) Imamura, A.; Kimura, A.; Ando, H.; Ishida, H.; Kiso, M. Extended applications of di-tert-butylsilylene-directed $\alpha$-predominant galactosylation compatible with C2-participating groups toward the assembly of various glycosides. Chem. - Eur. J. 2006, 12, 8862-8870. (51) Imamura, A.; Matsuzawa, N.; Sakai, S.; Udagawa, T.; Nakashima, S.; Ando, H.; Ishida, H.; Kiso, M. The Origin of High Stereoselectivity in Di-tert-butylsilylene-Directed $\alpha$-Galactosylation. J. Org. Chem. 2016, 81, 9086-9104.

(52) Schocker, N. S.; Portillo, S.; Brito, C. R.; Marques, A. F.; Almeida, I. C.; Michael, K. Synthesis of $\operatorname{Gal} \alpha(1,3) \mathrm{Gal} \beta(1,4)$ GlcNAc $\alpha$-, Gal $\beta(1,4)$ GlcNAc $\alpha$ - and GlcNAc-containing neoglycoproteins and their immunological evaluation in the context of Chagas disease. Glycobiology 2015, 26 (1), 39-50.

(53) Ashmus, R. A.; Schocker, N. S.; Cordero-Mendoza, Y.; Marques, A. F.; Monroy, E. Y.; Pardo, A.; Izquierdo, L.; Gállego, M.; Gascon, J.; Almeida, I. C.; Michael, K. Potential use of synthetic $\alpha$-galactosyl-containing glycotopes of the parasite Trypanosoma cruzi as diagnostic antigens for Chagas disease. Org. Biomol. Chem. 2013, 11 (34), 5579-5583.

(54) See the Supporting Information.

(55) Bai, Y.; Lowary, T. L. 2,3-Anhydrosugars in glycoside bond synthesis. Application to alpha-D-galactofuranosides. J. Org. Chem. 2006, 71 (26), 9658-9671.

(56) Completo, G. C.; Lowary, T. L. Synthesis of galactofuranosecontaining acceptor substrates for mycobacterial galactofuranosyltransferases. J. Org. Chem. 2008, 73 (12), 4513-4525.

(57) Deng, L. M.; Liu, X.; Liang, X. Y.; Yang, J. S. Regioselective glycosylation method using partially protected arabino- and galactofuranosyl thioglycosides as key glycosylating substrates and its application to one-pot synthesis of oligofuranoses. J. Org. Chem. 2012, 77 (7), 3025-3037.

(58) Winnik, F. M.; Carver, J. P.; Krepinsky, J. J. Syntheses of Model Oligosaccharides of Biological Significance. 2. Synthesis of a Tetramannoside and of Two Lyxose-Containing Trisaccharides. J. Org. Chem. 1982, 47, 2701-2707.

(59) Tilve, M. J.; Cori, C. R.; Gallo-Rodriguez, C. Regioselective 5O-Opening of Conformationally Locked 3,5-O-Di-tert-butylsilyleneD-galactofuranosides. Synthesis of $(1 \rightarrow 5)-\beta$-D-Galactofuranosyl Derivatives. J. Org. Chem. 2016, 81 (20), 9585-9594.

(60) Odiwuor, S. O.; Saad, A. A.; De Doncker, S.; Maes, I.; Laurent, T.; El Safi, S.; Mbuchi, M.; Buscher, P.; Dujardin, J. C.; Van der Auwera, G. Universal PCR assays for the differential detection of all
Old World Leishmania species. Eur. J. Clin. Microbiol. Infect. Dis. 2011, 30 (2), 209-218.

(61) Galili, U. Anti-Gal: an abundant human natural antibody of multiple pathogeneses and clinical benefits. Immunology 2013, 140 (1), $1-11$.

(62) Macher, B. A.; Galili, U. The Galalpha1,3Galbeta1,4GlcNAc-R (alpha-Gal) epitope: a carbohydrate of unique evolution and clinical relevance. Biochim. Biophys. Acta, Gen. Subj. 2008, 1780 (2), 75-88.

(63) Owino, B. O.; Matoke-Muhia, D.; Alraey, Y.; Mwangi, J. M.; Ingonga, J. M.; Ngumbi, P. M.; Casas-Sanchez, A.; Acosta-Serrano, A.; Masiga, D. K. Association of Phlebotomus guggisbergi with Leishmania major and Leishmania tropica in a complex transmission setting for cutaneous leishmaniasis in Gilgil, Nakuru county, Kenya. PLoS Neglected Trop. Dis. 2019, 13 (10), No. e0007712.

(64) Greiner, M.; Pfeiffer, D.; Smith, R. D. Principles and practical application of the receiver-operating characteristic analysis for diagnostic tests. Prev. Vet. Med. 2000, 45 (1-2), 23-41.

(65) El Safadi, D.; Merhabi, S.; Rafei, R.; Mallat, H.; Hamze, M.; Acosta-Serrano, A. Cutaneous leishmaniasis in north Lebanon: reemergence of an important neglected tropical disease. Trans. R. Soc. Trop. Med. Hyg. 2019, 113 (8), 471-476.

(66) Ozaras, R.; Leblebicioglu, H.; Sunbul, M.; Tabak, F.; Balkan, I. I.; Yemisen, M.; Sencan, I.; Ozturk, R. The Syrian conflict and infectious diseases. Expert Rev. Anti-Infect. Ther. 2016, 14 (6), 547555.

(67) Ready, P. D. Leishmaniasis emergence in Europe. Euro. Surveill. 2010, 15 (10), 19505.

(68) Torpiano, P.; Pace, D. Leishmaniasis: diagnostic issues in Europe. Expert Rev. Anti-Infect. Ther. 2015, 13 (9), 1123-1138.

(69) Schocker, N. S.; Portillo, S.; Ashmus, R. A.; Brito, C. R. N.; Silva, I. E.; Cordero-Mendoza, Y.; Marques, A. F.; Monroy, E. Y.; Pardo, A.; Izquierdo, L.; Gállego, M.; Gascon, J.; Almeida, I. C.; Michael, K. Probing for Trypanosoma cruzi cell surface glycobiomarkers for the diagnosis and follow-up of chemotherapy of Chagas disease. In Coupling and Decoupling of Diverse Molecular Units in Glycosciences; Witzczak, Z. J., Bielski, R., Eds.; Springer International Publishing AG: Cham, Switzerland, 2018; pp 195-211.

(70) Galili, U.; Rachmilewitz, E. A.; Peleg, A.; Flechner, I. A unique natural human IgG antibody with anti-alpha-galactosyl specificity. $J$. Exp. Med. 1984, 160 (5), 1519-1531.

(71) Naus, C. W.; van Remoortere, A.; Ouma, J. H.; Kimani, G.; Dunne, D. W.; Kamerling, J. P.; Deelder, A. M.; Hokke, C. H. Specific antibody responses to three schistosome-related carbohydrate structures in recently exposed immigrants and established residents in an area of Schistosoma mansoni endemicity. Infect. Immun. 2003, $71(10), 5676-5681$.

(72) Vermeer, H. J.; van Dam, G. J.; Halkes, K. M.; Kamerling, J. P.; Vliegenthart, J. F.; Hokke, C. H.; Deelder, A. M. Immunodiagnostically applicable monoclonal antibodies to the circulating anodic antigen of Schistosoma mansoni bind to small, defined oligosaccharide epitopes. Parasitol. Res. 2003, 90 (4), 330-336.

(73) Carvalho de Souza, A.; van Remoortere, A.; Hokke, C. H.; Deelder, A. M.; Vliegenthart, J. F.; Kamerling, J. P. Determination of the specificity of monoclonal antibodies against Schistosoma mansoni CAA glycoprotein antigen using neoglycoconjugate variants. Biol. Chem. 2005, 386 (9), 901-908.

(74) de Souza, L. M. B.; Thomaz Soccol, V.; Petterle, R. R.; Bates, M. D.; Bates, P. A. Analysis of Leishmania mimetic neoglycoproteins for the cutaneous leishmaniasis diagnosis. Parasitology 2018, 145 (14), $1938-1948$.

(75) Mandrekar, J. N. Receiver operating characteristic curve in diagnostic test assessment. J. Thorac. Oncol. 2010, 5 (9), 1315-1316.

(76) Zou, K. H.; O'Malley, A. J.; Mauri, L. Receiver-operating characteristic analysis for evaluating diagnostic tests and predictive models. Circulation 2007, 115 (5), 654-657.

(77) Ortega-Rodriguez, U.; Portillo, S.; Ashmus, R. A.; Duran, J. A.; Schocker, N. S.; Iniguez, E.; Montoya, A. L.; Zepeda, B. G.; Olivas, J. J.; Karimi, N. H.; Alonso-Padilla, J.; Izquierdo, L.; Pinazo, M. J.; de Noya, B. A.; Noya, O.; Maldonado, R. A.; Torrico, F.; Gascon, J.; 
Michael, K.; Almeida, I. C. Purification of Glycosylphosphatidylinositol-Anchored Mucins from Trypanosoma cruzi Trypomastigotes and Synthesis of alpha-Gal-Containing Neoglycoproteins: Application as Biomarkers for Reliable Diagnosis and Early Assessment of Chemotherapeutic Outcomes of Chagas Disease. Methods Mol. Biol. 2019, 1955, 287-308.

(78) Lopez, R.; Giorgi, M. E.; Melgarejo, L. T.; Ducrey, I.; Balouz, V.; Gonzalez-Salas, D.; Camara, M. L. M.; Buscaglia, C. A.; de Lederkremer, R. M.; Marino, C. Synthesis and characterization of alpha-d-Galp-(1->3)-beta-d-Galp epitope-containing neoglycoconjugates for chagas disease serodiagnosis. Carbohydr. Res. 2019, 478, 5867. 